\title{
Lengua, escuela e inmigración
}

\author{
IGNASI VILA* \\ Universidad de Girona

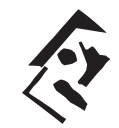 \\ Resumen
}

A lo largo de la última década, la heterogeneidad identitaria, lingüística, religiosa, cultural y étnica ha crecido de manera significativa en España debido a la llegada de inmigrantes, Lo cual está planteando al Sistema Educativo problemas hasta abora desconocidos. En este artículo se discuten las relaciones entre lengua, escuela e inmigración con un énfasis especial en los factores implicados en la adquisición de la lengua de uso escolar. Se discute tanto el tiempo que el alumnado inmigrante tarda en aprender dicha lengua como el papel de su lengua inicial en dicha adquisición. La discusión se realiza a partir del análisis de datos provenientes de evaluaciones internacionales, así como de trabajos de investigación realizados en situaciones escolares multilingüies y se reflexiona sobre su pertinencia para el establecimiento de una política educativa que tenga en cuenta las dificultades de las y los inmigrantes con la adquisición y el dominio de la lengua de la escuela. Finalmente, se abordan las nuevas necesidades de los sistemas educativos en relación con alguno de los retos que plantea la educación intercultural y se enfatiza la importancia de la práctica educativa para encontrar soluciones a los problemas que se derivan de la nueva situación escolar.

Palabras clave: Bilingüismo, plurilingüismo, inmigración, adquisición de segundas lenguas, escuela, atención a la diversidad.

\section{Language, school and immigration}

\begin{abstract}
During the last decade, linguistic, religious, cultural, ethnic and identity beterogeneity have significantly grown in Spain due to an increasing immigrants population. This is reflected in the educational system, which must confront problems hitherto unknown. In this article, we deal with the relationships between language, school and immigration, giving special emphasis to the factors involved in the acquisition of the language spoken at school. We discuss both the time it takes immigrant students to learn the school language and the role students' initial language has on its acquisition. The discussion is based on review data from international assessments and research work carried out in multilingual school settings. We reflect on its significance for establishing educational policies that take into account the difficulties immigrants have to face to acquire the school language. Finally, we address the new needs of educational systems in relation to some of the challenges posed by intercultural education, and we underline the importance of educational practice to find solutions to the problems that derive from the new situation in schools.
\end{abstract}

Keywords: Bilingualism, plurilingualism, immigration, second language learning, school, attention to diversity.

Agradecimientos: El trabajo ha sido posible gracias a una ayuda de la Dirección General de Investigación del Ministerio de Educación y Ciencia ( $\mathrm{n}^{\circ}$ referencia SEJ2005-08944-C02-01/EDUC).

Correspondencia con el autor: Universitat de Girona - Departament de Psicología C/ Creu, 217071 Girona. Tel.: 972-418254/E-mail: ignasi.vila@udg.es

Original recibido: Mayo, 2006. Aceptado: Junio, 2006. 


\section{Introducción}

La diversidad lingüística y cultural tiene una presencia cada vez mayor en el mundo occidental. España no es diferente y, a lo largo de la última década, la heterogeneidad identitaria, lingüística, cultural y étnica ha crecido de manera importante (Broeder y Mijares, 2003; Vila, 2005a). No cabe duda que ello se refleja en el sistema educativo que debe lidiar con problemas hasta ahora desconocidos más si se tiene en cuenta que numerosos trabajos manifiestan que el dominio de las habilidades lingüísticas implicadas en los procesos de enseñanza y aprendizaje es decisivo respecto al éxito o el fracaso escolar. Algunos de los problemas que se presentan son semejantes a los que la educación bilingüe ha intentado responder a lo largo del siglo XX, mientras que otros son claramente diferentes (Cummins, 2002; Vila, 2005a). En este artículo nos centramos exclusivamente en los aspectos relacionados con la adquisición de los inmigrantes de la lengua de la escuela. De hecho, una buena parte de estos y estas escolares se escolarizan en programas de cambio de lengua del hogar a la escuela y, con mayor o menor apoyo desde el entorno social, deben de desarrollar habilidades lingüísticas notables en la lengua de instrucción para poder participar activamente en las actividades de enseñanza y aprendizaje.

El éxito de los programas bilingües de inmersión lingüística es uno de los argumentos que se utilizan a menudo para justificar la política lingüística que se sigue con la escolarización de la infancia extranjera (Vila, 2005b). Así, se defiende que aquello que ha tenido éxito con el alumnado de habla castellana respecto al aprendizaje del catalán o del euskara o con el alumnado de habla inglesa respecto a la adquisición del francés, lo puede tener igual con el alumnado de hablas propias distintas de la lengua de la escuela y que como máximo, en relación con la infancia que se incorpora tardíamente a nuestro sistema educativo, se pueden utilizar "aulas-puente"1 o "aulas de adaptación lingüística" con el objetivo de que los alumnos aprendan los rudimentos de la lengua de la escuela y se incorporen activamente a ella y a sus actividades de enseñanza y aprendizaje.

Hemos organizado el artículo en cuatro apartados. En el primero, mostramos la diversidad lingüística que acompaña a la infancia y la adolescencia extranjera. En el segundo, mostramos alguna de las relaciones que existen entre los resultados escolares del alumnado extranjero y el dominio de la lengua de la escuela. En el tercero, abordamos específicamente los factores implicados en la adquisición de la lengua de la escuela y, finalmente, discutimos la validez de la tradición de homogeneidad de la práctica educativa para encarar con éxito la actual diversidad lingüística en el contexto escolar.

\section{Algunos datos sobre la infancia extranjera: escolarización y lengua propia}

El fenómeno migratorio de la última década ha transformado radicalmente la situación lingüística de las aulas de una buena parte de los sistemas educativos del Estado Español. En concreto, en la Comunidad de Madrid, en el Levante español, en La Rioja, en Navarra, en Cataluña y en las Islas Canarias -sin olvidar las ciudades autónomas de Ceuta y Melilla y, en menor medida, Andalucía-, la realidad lingüística de las aulas se ha visto profundamente modificada. Por ejemplo, Broeder y Mijares (2003) muestran que en las aulas de la Comunidad de Madrid junto al español hay cerca de 50 lenguas diferentes utilizadas en casa bien solas, bien junto al español, que se corresponde con el 10\% de la población encuestada. Del conjunto de lenguas citadas, sólo 8 lo fueron por más de 100 alumnos y alumnas (árabe, ingles, portugués, chino, francés, rumano, tagalo y polaco). En Cataluña, un estudio reciente del Grup de Llengües Amenaçades del 
Departament de Lingüística General de la Universitat de Barcelona se cifra en más de 200 el número de lenguas presentes (cit. en Nadal, 2006) y si nos atenemos al número de lenguas del alumnado de la educación primaria y la educación secundaria, algo más de 12.000 alumnos y alumnas, que fue atendido en las aulas de acogida durante el curso 2004-2005 encontramos más de 30 lenguas, de las cuales 4 (árabe, amazit, chino, rumano) eran habladas por más de 500 alumnos. En el resto de comunidades con una importante presencia de personas inmigrantes las cosas no son muy distintas. Además, existen dos factores que nos hacen pensar que en el futuro cercano las cosas no se van a modificar. Así, primero, las familias extranjeras tienden a mantener en el hogar su propia lengua y, segundo, una característica importante de estas personas es que en un porcentaje importante son mujeres jóvenes (Ajuntament de Barcelona, 2005; Izquierdo, 2002) lo cual significa que una parte, cada vez más importante, de los niños y niñas nacidos en nuestro país tendrá como lengua inicial una lengua distinta de la lengua de la escuela. Sin duda, la falta de estudios hace difícil determinar el porcentaje de alumnado que tiene como lengua propia una lengua diferente del español o de la lengua oficial de la comunidad, pero no es menos cierto que estudios parciales (Broeder y Mijares, 2003) muestran que crece en proporción geométrica y que, por ejemplo, en algunas zonas de Cataluña supera el 30\% del conjunto del alumnado.

Esta situación es completamente distinta de las realidades que han suscitado muchas de las investigaciones y las reflexiones de la educación bilingüe. Así, de manera general, la enseñanza bilingüe se ha preocupado principalmente de organizar la educación para promover conocimiento de una segunda lengua desde la escuela a grupos lingüísticamente homogéneos: enseñanza del francés a alumnado de habla inglesa, enseñanza del inglés a alumnado de habla hopi, enseñanza del catalán a alumnado de habla castellana y semejantes. De hecho, la educación bilingüe ha compartido con el resto de la educación la idea de que "cuanto más homogénea sea una situación, más posibilidades de promover éxito académico desde la práctica educativa". Por eso, todos los modelos de educación bilingüe conocidos como exitosos siempre han buscado la homogeneidad lingüística de su alumnado respecto a la lengua de la escuela, bien mediante su coincidencia (programas de mantenimiento de la lengua familiar), bien mediante su desconocimiento (programas de inmersión lingüística). Sin embargo, la situación descrita anteriormente comporta concreciones muy diferentes en nuestras aulas. En resumen, podemos destacar tres. Una, en la que una parte del alumnado, más o menos grande, pero siempre significativa, es lingüísticamente heterogénea y comparte su escolarización con otra parte de alumnos nacionales que tienen como lengua propia la lengua de la escuela. Dos, una situación semejante a la anterior pero en la que el alumnado nacional es de habla castellana y sigue un programa de inmersión lingüística de modo que la lengua de la escuela y la lengua del alumnado no coincide en ningún caso y, tres, una situación en la que más del $80 \%$ del alumnado es extranjero y muestra una enorme diversidad lingüística de modo que en las aulas puede haber 6.7 o más lenguas diferentes y, en el conjunto de la escuela, entre 13, 14, 15 o más lenguas (Vila y Siqués, 2006a, en prensa).

Por eso, como mínimo es discutible realizar una transposición mecánica de algunos de los presupuestos de la educación bilingüe a la comprensión y la organización lingüística de nuestra realidad educativa.

\section{Inmigración, resultados escolares y lengua de escuela}

Aún existen pocas investigaciones en nuestro país que traten las relaciones entre el conocimiento de la lengua de la escuela, de la propia lengua y el rendi- 
miento escolar. Por eso, es aventurado extraer conclusiones generales de los trabajos existentes ya que la diversidad de la infancia extranjera es enorme desde muchos puntos de vista: conocimiento de su propia lengua, nivel de escolarización previo, momento de incorporación a nuestro sistema educativo, nivel educativo de la familia y expectativas educativas, actitudes hacia las lenguas y semejantes. No obstante, los trabajos existentes sobre la relación entre inmigración y éxito escolar son decepcionantes. Recientemente, INECSE (2006) ha publicado los resultados de la evaluación realizada en 2003 en $6^{\circ}$ de Primaria acerca del conocimiento del medio, matemáticas y lengua castellana. La figura 1 muestra los resultados según las características lingüísticas de los progenitores y el hecho de ser o no extranjeros en las comunidades bilingües y monolingües.

FIGURA 1

Diferencias en el rendimiento en función de la situación lingüística de la familia. Sexto de Educación Primaria. Curso 2002-2003

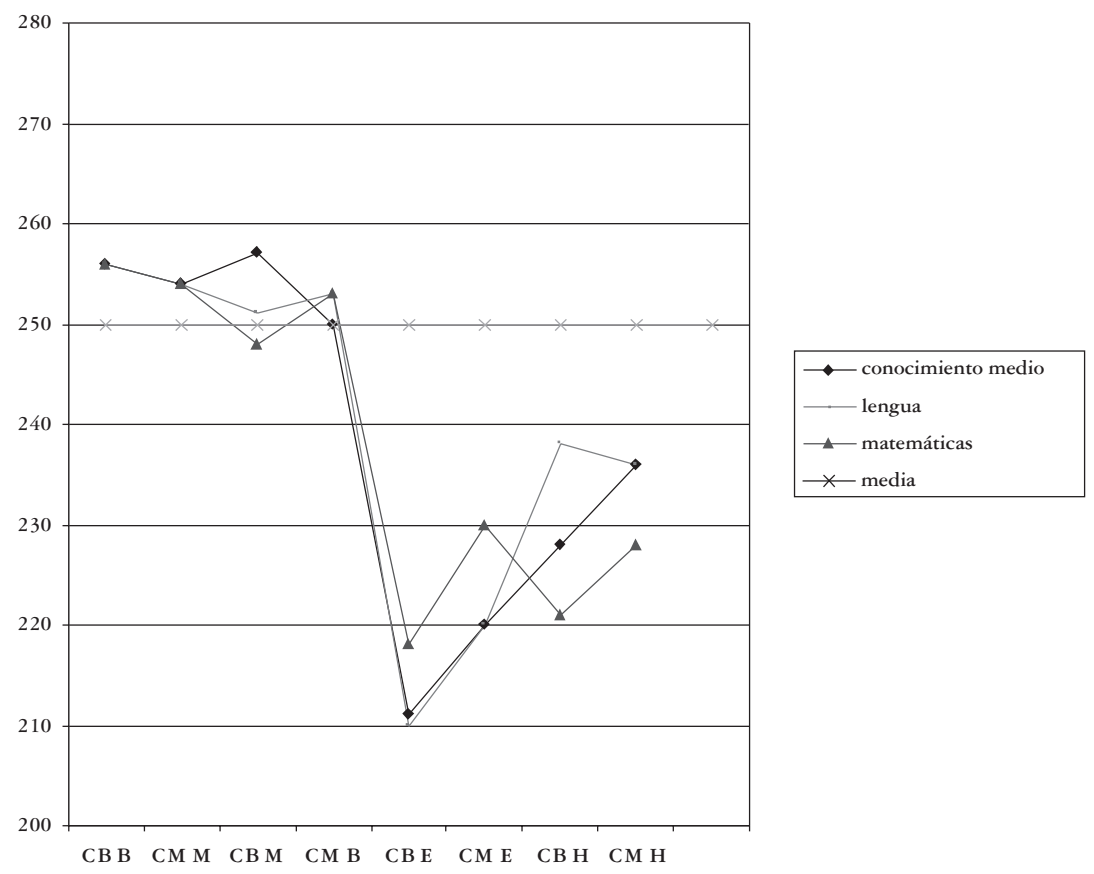

CB: comunidad bilingüe; CM: comunidad monolingüe; B: progenitores bilingües; M: progenitores monolingües; E: progenitores extranjeros; $\mathrm{H}$ : progenitores hispanos. Fuente: INECSE (2006).

En algunas categorías, el alumnado evaluado es muy escaso y, por eso, como afirman los autores del trabajo no pueden establecerse diferencias significativas. Ferrer, Ferrer y Castel (2006) han utilizado la base de datos PISA 2003 para analizar las desigualdades en Cataluña. La figura 2 muestra los resultados según el origen del alumnado.

La muestra utilizada bajo el epígrafe "alumnado no nativo" es también pequeña $(4,7 \%)$ al igual que la muestra de "extranjeros" en el estudio de la Evaluación Primaria en 2003. No obstante, todos los datos, al igual que en la mayoría de los países de la $\mathrm{OCDE}^{2}$, apuntan a un rendimiento académico más bajo del alumnado inmigrante. 
FIGURA 2

Rendimiento académico y origen del alumnado. 15 años. Cataluña
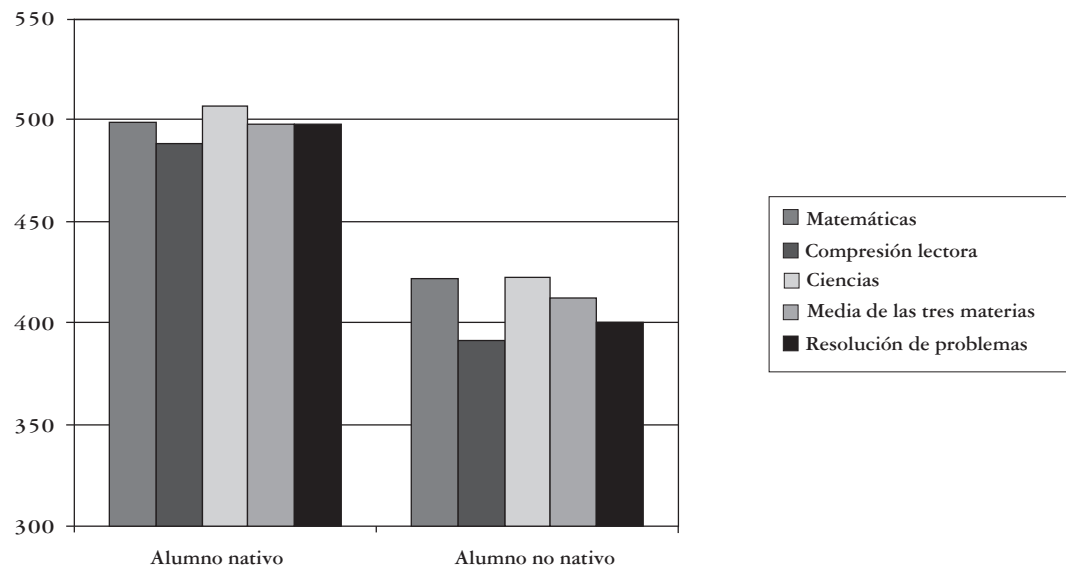

El alumnado nativo incluye también el alumnado no nativo de primera generación. Fuente: Ferrer et al. (2006).

Una de las explicaciones de esta realidad se corresponde con su nivel lingüístico o, en otras palabras, con la falta de habilidades respecto a la lengua que emplea la escuela en las actividades de enseñanza y aprendizaje. De hecho, tanto Siguan (1998) como Fullana, Besalú y Vilà (2003) señalan esta cuestión como decisiva. Así, ambas investigaciones muestran que las calificaciones en la lengua de la escuela de los escolares extranjeros eran significativamente más bajas que las de sus pares autóctonos. En este sentido, existen otros trabajos (Areny y Vial, 2005; Maruny y Molina, 2000; Navarro y Huguet, 2005; Vila y Siqués, 2006a, en prensa) que se han preocupado especialmente en conocer la competencia lingüística de dichos escolares respecto a la lengua de la escuela.

Maruny y Molina (2000) en un estudio realizado con niños y niñas marroquíes escolarizados entre $3^{\circ}$ de Primaria y $4^{\circ}$ de ESO en la comarca gerundense del Baix Empordà muestran que hace falta tres años de escolarización para desarrollar suficiente competencia conversacional en la lengua de la escuela, cinco años en la escuela para mostrar una comprensión lectora aceptable y, en relación con la escritura, no hubo ningún sujeto de la muestra que tuviera un nivel adecuado. Además, no aparecen diferencias significativas entre el alumnado nacido en la comarca y el que se incorpora tardíamente.

Navarro y Huguet (2005) estudian la competencia lingüística de los escolares procedentes de la inmigración extracomunitaria escolarizados en $1^{\circ}$ de ESO en la provincia de Huesca a lo largo del curso 2002-2003. Su muestra fue de 93 alumnos de los cuales 49 eran inmigrantes de incorporación tardía y 44 nativos. Entre los distintos indicadores empleados, dicha investigación utiliza los índices PG1 y PG2 en el que el primero está formado por subpruebas de comprensión oral, morfosintaxis, ortografía, comprensión escrita y expresión escrita, mientras que el segundo contempla las anteriores más la organización de la información, la fonética, la corrección lectora y la entonación lectora. En su trabajo se compara la competencia del alumnado inmigrante en ambos índices con la de sus iguales autóctonos. La tabla I muestra los resultados. 
TABLA I

Comparación de los índices PG1 y PG2 de los escolares inmigrantes y autóctonos en Primero de ESO de la provincia de Huesca

\begin{tabular}{lcl}
\hline & PG1 & PG2 \\
\hline INMIGRANTES & 58,26 & 59,64 \\
AUTÓCTONOS & 77,76 & 77,15 \\
\hline
\end{tabular}

Fuente: Navarro y Huguet (2005).

Además, Navarro y Huguet (2005) muestran que dicha diferencia se mantiene independientemente de la lengua propia del alumnado. Es decir, el alumnado de habla hispana también obtiene peores resultados que el alumnado autóctono aunque mejor que los que tienen como lengua propia una lengua distinta del español. La tabla II muestra la existencia o ausencia de diferencias significativas entre los diferentes grupos lingüísticos y el grupo de los autóctonos.

TABLA II

Significatividad de las diferencias entre los diferentes grupos lingüisticos y el grupo de los autóctonos en Primero de ESO en la provincia de Huesca

\begin{tabular}{lrr}
\hline & PG1 & PG2 \\
\hline SÍ HISPANO VERSUS NO HISPANO & $0,0079 *$ & $0,0226^{*}$ \\
SÍ HISPANO VERSUS AUTÓCTONO & $0,0039^{*}$ & 0,1106 \\
NO HISPANO VERSUS AUTÓCTONO & $<0,0001 *$ & $0,0006^{*}$ \\
\hline
\end{tabular}

Fuente: Navarro y Huguet (2005)

Todas las diferencias son significativas excepto, en relación con el PG2, entre el grupo de los hispanos y los autóctonos. Sin embargo, a pesar de la falta de significación, las medias de cada grupo eran respectivamente de 67,209 y 77,145.

Areny y Vial (2004) analizan el conocimiento de catalán en Segundo de Primaria el Curso 1998-1999 en una muestra de 44 escuelas y 850 alumnos de los cuales 177 eran catalanohablantes y 208 tenían como lengua familiar lenguas diferentes del catalán y del castellano. De los 208 alumnos extranjeros, 45 eran de incorporación tardía y se habían incorporado a la escuela después de P-4. Todas las escuelas hacían la enseñanza en catalán. La tabla III muestra los resultados para los índices PG1 y PG2.

TABLA III

Conocimiento de catalán del alumnado extranjero y el alumnado catalanobablante escolarizado en lengua catalana. Segundo de Primaria. Curso 1998-1999

\begin{tabular}{lcl}
\hline & PG1 & PG2 \\
\hline Alumnado catalanohablante & 76,96 & 79,71 \\
\hline Alumnado extranjero & 59,32 & 64,04 \\
\hline
\end{tabular}

Fuente: Areny y Vial (2005).

Areny y Vial (2005) afirman que 123 alumnos extranjeros tienen resultados muy bajos en lengua catalana y señalan que, de ellos, 45 son de incorporación tardía y, excepto en 8 escuelas, el alumnado extranjero tiene resultados inferiores al alumnado de lengua familiar catalana y de lengua familiar castellana. 
Por último, Vila y Siqués (2006a, en prensa), en un seguimiento etnográfico longitudinal realizado durante dos años en un aula al inicio de la educación primaria formada fundamentalmente por inmigrantes extracomunitarios, muestran que el uso de la lengua de la escuela que realizan los alumnos, independientemente del momento de su escolarización, se asemeja al que realizan los autóctonos de edad semejante en las relaciones cara-a-cara altamente contextualizadas y que, además, tienen un conocimiento muy pobre del léxico que se emplea en las actividades de enseñanza y aprendizaje.

En el resto de los países occidentales, las cosas no son muy distintas. El fenómeno migratorio ligado a la globalización alcanza a la inmensa mayoría de los países de la OCDE. Así, los problemas lingüísticos del alumnado extranjero alcanzan a todos los sistemas educativos y no únicamente a los del Estado Español. Tras la evaluación PISA 2002 sobre habilidades lectoras del alumnado de 15 años, el informe de la OCDE afirmaba:

En varios países, los estudiantes extranjeros tienen un rendimiento en habilidad lectora mucho menor que el promedio de los estudiantes que nacieron (así como sus padres) en el país. Obtienen puntuaciones, por lo menos, 71 puntos inferiores a las de los nativos en 10 de los 15 países con más del $3 \%$ de población inmigrante, aunque en algunos países esta diferencia es mucho menor (OCDE, 2002, p. 27).

Es decir, el alumnado inmigrante en la gran mayoría de países ${ }^{3}$ del mundo occidental tiene problemas con la lengua de la escuela y tarda tiempo en solventarlos.

\section{El alumnado extranjero y la adquisición de la lengua de la escuela}

La adquisición del alumnado extranjero de las habilidades lingüísticas implicadas en las actividades de enseñanza y aprendizaje es un proceso largo y complejo. Cummins (2002) lo expresa de la siguiente forma:

Los estudios de investigación desde principio de los años ochenta muestran que los estudiantes inmigrantes pueden adquirir rápidamente una fluidez considerable en la lengua dominante de la sociedad cuando están expuestos a la misma en el entorno y en la escuela. Sin embargo, pese a este rápido progreso en fluidez conversacional generalmente hace falta un mínimo de cinco años (y a menudo muchos más) para recuperar el terreno con los hablantes nativos en los aspectos académicos de la lengua (Cummins, 2002, p. 50).

Los datos empíricos que apoyan esta afirmación provienen tanto de la evaluación de los programas bilingües de inmersión lingüística como de los obtenidos con el alumnado extranjero de incorporación tardía.

En 1990, en Cataluña, se realizó una evaluación de la inmersión lingüística en $6^{\circ}$ de Primaria en una muestra representativa de dichas escuelas (Bel, Serra y Vila, 1992). Los resultados sobre conocimiento de catalán se compararon con una muestra representativa de escuelas catalanas que escolarizaban fundamentalmente a alumnos de lengua familiar catalana. Las tablas IV y V muestran los resultados para los índices PG1 y PG2 según el nivel socio-profesional de las familias.

Los datos muestran que, después de seis años de educación obligatoria, el alumnado de inmersión lingüística es menos competente en la lengua de la escuela que sus pares que la tienen como lengua inicial. Esta constatación es conocida desde la extensión de los programas de inmersión lingüística y lleva a autores como McLaughlin a afirmar en relación con los programas de inmersión lingüística en Canadá: "Así, parece que incluso después de 6 o 7 años, el francés de los estudiantes de inmersión no es como el de los nativos” (McLaughlin, 1985 , p. 64). 
TABLA IV

Comparación entre el alumnado de inmersión lingüística y el alumnado catalanobablante escolarizado en catalán sobre competencia en lengua catalana escrita y nivel socioprofesional de las familias. Sexto de EGB. Curso 1989-1990

\begin{tabular}{|c|c|c|c|c|c|c|}
\hline \multirow{3}{*}{ Nivel Socioprofesional } & \multicolumn{6}{|l|}{ PG1 } \\
\hline & \multicolumn{2}{|l|}{ Alto } & \multicolumn{2}{|l|}{ Medio } & \multicolumn{2}{|l|}{ Bajo } \\
\hline & $X$ & $S$ & $X$ & $S$ & $X$ & $S$ \\
\hline Alumnado de inmersión lingüística & 57,52 & 16,98 & 56,26 & 14,02 & 54,46 & 15,94 \\
\hline $\begin{array}{l}\text { Alumnado de lengua catalana } \\
\text { escolarizado en catalán }\end{array}$ & 68,28 & 13,47 & 68,87 & 12,22 & 67,12 & 12,69 \\
\hline
\end{tabular}

$X=$ media y $S=$ desviación estándar.

Fuente: Bel, Serra i Vila (1992).

TABLA V

Comparación entre el alumnado de inmersión lingüística y el alumnado catalanohablante escolarizado en catalán sobre competencia en lengua catalana escrita y oral y nivel socioprofesional de las familias.

Sexto de EGB. Curso 1989-1990

\begin{tabular}{|c|c|c|c|c|c|c|}
\hline \multirow{3}{*}{ Nivel Socioprofesional } & \multicolumn{6}{|l|}{ PG2 } \\
\hline & \multicolumn{2}{|l|}{ Alto } & \multicolumn{2}{|l|}{ Medio } & \multicolumn{2}{|l|}{ Bajo } \\
\hline & $X$ & $S$ & $X$ & $S$ & $X$ & $S$ \\
\hline Alumnado de inmersión lingüística & 60,30 & 8,90 & 63,07 & 13,65 & 60,13 & 15,51 \\
\hline $\begin{array}{l}\text { Alumnado de lengua catalana } \\
\text { escolarizado en catalán }\end{array}$ & 77,39 & 8,02 & 75,30 & 10,68 & s.d. & s.d. \\
\hline
\end{tabular}

$X=$ media y $S=$ desviación estándar.

Fuente: Bel, Serra i Vila (1992).

Las implicaciones para la práctica educativa y la organización escolar sobre el tiempo que tarda el alumnado de los programas, bien diseñados, de cambio de lengua del hogar a la escuela en equiparse al conocimiento de la lengua de la escuela que tienen sus pares monolingües son muchas y diversas. Una de ellas, ya referida anteriormente, hace mención al mantenimiento del máximo grado de homogeneidad del alumnado respecto al conocimiento de la lengua de la escuela. Es decir, la eliminación de mezclas en la misma aula de alumnado cuya lengua coincide con la lengua de la escuela y alumnado con una lengua diferente a la lengua de la escuela.

Pero, en relación con nuestra discusión, estos datos plantean una pregunta importante respecto a la escolarización de la infancia y la adolescencia extranjera: si el alumnado de inmersión lingüística tarda más de 6 años en equiparar su conocimiento de la lengua de la escuela al de sus pares monolingües, ¿cuánto tiempo tarda un alumno o una alumna inmigrante de incorporación tardía en equiparar su conocimiento de la lengua de la escuela al del alumnado nativo? Y una pregunta más, ¿y el alumnado extranjero, nacido en el país de acogida, que mantiene en su casa la lengua familiar, cuánto tarda en desarrollar un buen conocimiento de la lengua de la escuela y en qué condiciones?

Un buen ejemplo es Canadá , país con un elevado número de personas inmigrantes y de alumnado extranjero en su sistema educativo. Los resultados del sistema educativo de Canadá en las diferentes evaluaciones internacionales son excelentes y, además, la diferencia de puntuación entre el alumnado extranjero y 
el alumnado nacional es muy pequeña. La figura 3 muestra el tiempo que tarda el alumnado extranjero en Canadá en equiparse en conocimiento de la lengua de la escuela, medido mediante las habilidades de lectura, al de sus pares nacionales.

FIGURA 3

Competencia del alumnado extranjero de Canadá en lectura y años de residencia en Canadá. 15 años. PISA 2000

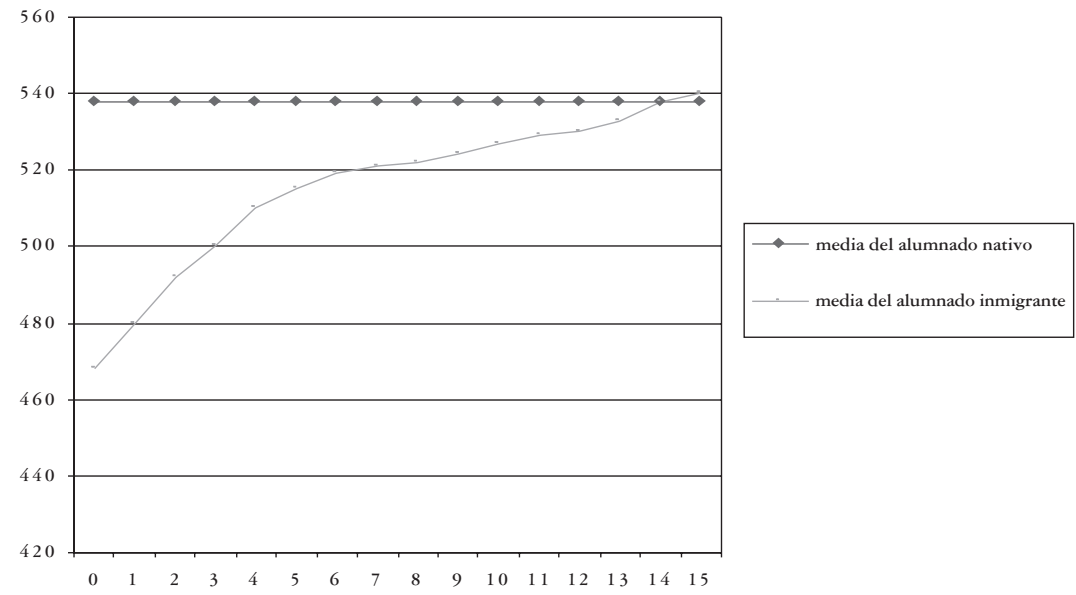

Fuente: Statistics Canada (http://www.statcan.ca/).

Cummins (1981) informa, tras un estudio con 1.210 inmigrantes que llegaron a Canadá a la edad de 6 años y que se escolarizaron en lengua inglesa, que, por término medio, este alumnado tarda entre 5 y 7 años para adquirir competencia en las habilidades en lengua inglesa que requiere la escuela semejante a sus pares anglófonos a pesar de que, después de dos o tres años, mostraban una gran fluidez oral en actividades conversacionales.

Tras este estudio pionero, se han sucedido una gran número de trabajos (Collier, 1987; Gandara, 1999; Hakuta, Butler y Witt, 2000; Huguet y Navarro, 2005; Klesmer, 1994; Maruny y Molina, 2000; Navarro y Huguet, 2005; Ramírez, 1992; Shohamy, 1999; Thomas y Collier, 1997; Vila, Huguet y Navarro, 2005; Vila y Siqués, 2006b) que apoyan que el alumnado extranjero tarda entre 6 y 12 años, según las condiciones de escolarización, en incorporar conocimiento de la lengua de la escuela semejante al de sus pares anglófonos nativos.

El estudio de Thomas y Collier (1997) tiene un especial interés. Su trabajo resume los resultados de su investigación realizada entre 1982 y 1996 en cinco sistemas escolares de Estados Unidos en los que coexistían diferentes programas, desde la enseñanza únicamente en inglés hasta programas bilingües en dos lenguas, y que agrupaban alrededor de 700.000 estudiantes de lengua inicial distinta al inglés. Las evaluaciones se realizaban longitudinalmente (por ejemplo, 42.317 escolares fueron evaluados a lo largo de 4 o más años) y transversalmente. El número de lenguas presentes era 150 y la lengua mayoritaria era el español (63\%). Los datos incluyen todos los tipos posibles de "extranjería": nuevos inmigrantes, bien de incorporación tardía, bien nacidos en USA; grupos de refugiados políticos de distintas partes del mundo y alumnado de segunda y tercera generación que mantiene su lengua familiar en el hogar. 
Sus resultados muestran que el alumnado inmigrante, escolarizado únicamente en inglés, que llegaba a los Estados Unidos en edades entre 8 y 11 años y que habían estado previamente escolarizados al menos entre 2 y 5 años en su lengua familiar tardaba entre 5 y 7 años en equipararse en conocimiento de la lengua de la escuela a sus pares nativos. El alumnado que llegaba antes de los 8 años requería entre 7 y 10 años o más en realizar dicha equiparación y el que llegaba después de los 12 años con una buena escolarización en su lengua familiar no llegaba nunca a atrapar a sus pares anglófonos nativos.

A la vez, el autor y la autora afirman que los factores más importantes que predicen el éxito académico de estos y estas escolares son la escolarización previa en su lengua inicial y la asistencia a programas escolares en los que una parte de la enseñanza se realiza en la lengua familiar del alumnado. A ambos factores, en dos distritos escolares de los cinco estudiados, se añade el nivel educativo de las familias.

La importancia del tratamiento educativo de la lengua inicial del alumnado extranjero, bien en su escolarización previa, bien en el programa educativo del país de acogida (Coelho, 1998, 2003; Vila, 2000) se explica mediante la hipótesis de interdependencia lingüística formulada, en 1979, por Cummins (1979, 2001), según la cual el desarrollo de habilidades lingüísticas en la propia lengua ayuda a la adquisición de nuevas habilidades desde la segunda lengua que, si se dan las condiciones adecuadas, pueden transferirse a la propia lengua.

Tal y como explican Thomas y Collier (1997), la razón por la cual tanto el alumnado de inmersión lingüística como el alumnado extranjero de incorporación tardía tarda tanto tiempo en equiparar su conocimiento de la lengua de la escuela al alumnado nativo remite a la idea de que el alumnado nativo no "espera” al alumnado cuya lengua inicial no coincide con la lengua escolar. Es decir, el alumnado nativo está en un entorno social y familiar que promueve competencias lingüísticas en su lengua inicial, las cuales coinciden con las que requiere la escuela, la cual, a su vez, promueve nuevas competencias lingüísticas en dicha lengua, mientras que el alumnado que participa en un programa de cambio de lengua del hogar a la escuela debe de aprender, en primer lugar, a hacer en la nueva lengua aquello que ya hace en su lengua, lo cual conlleva tiempo. En segundo lugar, el entorno social y familiar no siempre favorece, en estos casos, el desarrollo de competencias lingüísticas en la lengua de la escuela y, en tercer lugar, en numerosas ocasiones, este alumnado está mezclado con alumnado nativo o con alumnado extranjero que lleva años escolarizado en la lengua de la escuela de modo que el input lingüístico que recibe no es comprensible (Krashen, 1985) con sus consecuencias negativas para su desarrollo lingüístico.

¿Y en el caso del alumnado hispano en España? Ciertamente, hay pocas investigaciones que aborden el desarrollo de las habilidades lingüísticas escolares del alumnado hispano, pero las que existen muestran que también este alumnado tarda años en equiparar su conocimiento de la lengua de la escuela al alumnado nativo monolingüe. Un ejemplo remite a la evaluación de las competencias básicas en lengua castellana en $6^{\circ}$ de Primaria (INECSE, 2006), antes mencionado, según el cual este alumnado sabe menos lengua castellana al finalizar la enseñanza primaria que sus pares castellano-hablantes tanto en las comunidades monolingües como en las bilingües.

Vila et al. (2005) utilizan los datos de la investigación de Navarro y Huguet (2005) para analizar el tiempo que tarda el alumnado hispano en equiparar su conocimiento de la lengua de la escuela a sus pares nativos.

La tabla VI muestra, primero, que a más tiempo de residencia del alumnado extranjero más conocimiento de la lengua de la escuela y, segundo, el alumnado hispano en todas las condiciones tiene más competencia en la lengua de la escue- 
TABLA VI

Comparación del conocimiento de castellano del alumnado inmigrante bispano y no bispano de Huesca según el tiempo de residencia. $1^{\circ}$ de ESO. Curso 2002-2003

\begin{tabular}{lllll}
\hline Tiempo de residencia & PG1 & & PG2 & \\
\hline & Hispano & No hispano & Hispano & No hispano \\
Menos de 3 años & 65,56 & 49,66 & 65,69 & 50,14 \\
Entre 3 y 6 años & 66,82 & 59,33 & 72,18 & 62,48 \\
Más de 6 años & 68,41 & 68,97 & 70,96 & 72,96 \\
\hline
\end{tabular}

Fuente: Vila, Huguet y Navarro (2005).

la que el alumnado extranjero no hispano. Sin embargo, la comparación entre el alumnado hispano y el alumnado nativo muestra que no hay diferencias significativas entre ellos si el tiempo de residencia es de 6 o más años, pero existen diferencias significativas en comprensión oral y expresión escrita si el tiempo de residencia es de 3 a 6 años y, en relación a los que llevan menos de 3 años, las diferencias son significativas tanto para el PG1 como para el PG2.

Estas constataciones comportan que Hakuta et al. (2000) afirmen en relación con las "aulas-puente" en Estados Unidos:

Los datos sugieren que las políticas que asumen la adquisición rápida del inglés -el caso extremos es la proposición 227 que explícitamente afirma la necesidad de "proteger de la inmersión en inglés durante un periodo temporalmente transitorio que normalmente no exceda de un año"-son de una falta de realismo sorprendente. Una política mucho más sensible sería aquella que desechara el espectro total de los grados elementales como el ámbito en el que se consigue adquisición del inglés, y que planificara un currículum balanceado que prestara atención no únicamente al inglés, sino al conjunto de necesidades académicas de los estudiantes (Hakuta $e t$ al., 2000, pp. 13-14).

O que autores como Cummins clamen contra las políticas que consideran que, después de dos años, el alumnado extranjero ya conoce la lengua de la escuela.

¿De dónde ha salido, entonces, el número de dos años como criterio para incluir o excluir de las
pruebas nacionales a los alumnos que estudian inglés como segunda lengua? Es muy posible
que se derive de la idea de "sentido común" de que éste es el tiempo que tardan los estudiantes
en "aprender inglés"....Esta concepción de la naturaleza del dominio del inglés es ingenua o,
quizá, cínica; refleja los típicos períodos necesarios para adquirir un grado razonable de fluidez
conversacional en inglés, pero no el tiempo preciso para alcanzar a quienes tienen el inglés
como lengua materna en aspectos académicos del idioma. Este ejemplo y muchos más que
podríamos tomar de otros contextos revelan la importancia de que los responsables políticos y
los educadores tengan una concepción clara de lo que pretenden decir con la expresión "domi-
nio del idioma". En una época de diversidad lingüística y cultural generalizada, es probable
que las políticas educativas sobre el currículum y la evaluación, que relegan las consideraciones
relativas a la diversidad y al lenguaje al nivel de "anexo" o de "nota a pie de página", produzcan
una enseñanza discriminatoria y unos datos de rendición de cuentas carentes de sentido (Cum-
mins, 2002, pp. 30-31).

Esta constatación, junto con la idea de la escuela inclusiva, ha modificado la atención lingüística al alumnado extranjero en los países con mejores resultados en las evaluaciones internacionales. Resulta curioso que, en España, tras las evaluaciones PISA 2000 y 2003, una buena parte de los responsables educativos explicaran que una de las razones del éxito de Finlandia se relacionaba, entre otras razones, con la falta de inmigración y, sin embargo, no hubiera ni una sola declaración que explicara las razones por las que, Canadá, con un número de niños y niñas extranjeros mucho más elevado que en España, se disputa con Finlandia, en todas las evaluaciones internacionales, los primeros puestos en el ranking (Ferrer et al., 2006). 
Ya, en 1969, en Québec (Canadá), se inician “aulas de acogida” cerradas dirigidas a la inmigración de incorporación tardía que tenían como objetivo el aprendizaje de la lengua de la escuela. Estas aulas tienen una ratio especial, entre 15 y 20 alumnos y alumnas, un profesorado especializado en la enseñanza de la lengua de la escuela y, además, en ellas, junto con el aprendizaje del francés se pretende garantizar también otros aprendizajes del currículum, si bien lo que centra fundamentalmente su actividad es el aprendizaje del francés. En 1984, se extiende el programa cuyo objetivo era el inicio del aprendizaje de la lengua francesa, lengua de la escuela, que debía completarse a lo largo de la escolaridad ordinaria. Una vez en el aula ordinaria, el alumnado alófono tiene apoyos lingüísticos durante dos años. Sin embargo, tanto las comisiones de escolarización como los propios sindicatos, presionaron durante años para que la estancia del alumnado inmigrante en estas aulas se prolongara en el tiempo, de modo que, en 1988, cerca del $30 \%$ del alumnado extranjero pasaba en dichas aulas dos o más años y en el año 2000 alcanzaba al 50\% (Mc Andrew, 2001). A pesar de ello, "los enseñantes de las clases ordinarias parecen considerar que un porcentaje importante de alumnos de las clases de acogida integran sus clases insuficientemente preparados y que su tarea se hace cada vez más pesada año tras año" (Mc Andrew, 2001, p. 30). Las evaluaciones muestran que, a pesar del coste de un aprendizaje intensivo de la lengua francesa en un contexto cerrado e independiente del aula ordinaria, un 37,6\% del alumnado de la educación primaria sigue una escolaridad normal y el resto presenta únicamente un año de retraso, el año dedicado al aprendizaje de la lengua. Respecto al alumnado que llega a la educación secundaria, los resultados son distintos. Así, el 48,4\% de los que llegan con una escolarización deficiente en su lengua propia presentan un retraso de dos o más años y el $337 \%$ no obtienen el certificado al finalizar la enseñanza obligatoria. Finalmente, el alumnado alófono en los exámenes nacionales obtiene resultados sólo algo peor que el alumnado nativo. 57\%, 66\% y $69 \%$ (escritura, lectura y lengua oral) frente al 64\%, $71 \%$ y $73 \%$ (Mc Andrew, 2002).

Sin embargo, a partir del año 1998, Québec se ha cuestionado este modelo, especialmente para el alumnado de la educación primaria, tras interrogarse, a la vez, por el valor de la homogeneidad en el sistema educativo. Así, la evolución de la inmigración tanto desde el punto de vista numérico como desde el punto de vista de su diversidad ha cuestionado el modelo de "clase cerrada" y se han ensayado con fortuna otras fórmulas como son la combinación del "aula de acogida" y el "aula ordinaria” o la incorporación desde el inicio al aula ordinaria. En ambos casos, se considera que el lugar principal para el aprendizaje de la lengua de la escuela es el aula ordinaria y que, para hacerlo posible, se debe de buscar apoyos humanos y organizativos al trabajo del profesorado en el aula ordinaria. En concreto, al igual que ocurre en el País Vasco, en estos modelos se incorpora profesorado de apoyo lingüístico al alumnado extranjero en el aula ordinaria (Fortín, 2006). De hecho, Québec sigue el modelo del Canadá inglés, especialmente el de las ciudades de Toronto y Vancouver, en las que el alumnado extranjero se incorpora desde el primer momento al aula ordinaria y recibe un apoyo lingüístico específico en el aula durante, por ejemplo, en Toronto, cuatro años como mínimo, junto con clases específicas de lengua de la escuela durante unas horas a la semana.

De hecho, tal y como ha ocurrido en Cataluña en los dos últimos años, este último modelo es el que se va generalizando en los sistemas educativos occidentales con un elevado porcentaje de alumnado extranjero para dar una respuesta educativa adecuada a la diversidad lingüística y cultural creciente. No obstante, queda sin resolver la atención lingüística al alumnado de incorporación tardía a la educación secundaria con una escolarización previa irregular. En esta situa- 
ción, el modelo mayoritario que se emplea es el aula cerrada en la que, junto con los rudimentos de la lengua se enseñan otros aspectos instrumentales del currículum como, por ejemplo, la matemática.

\section{Inmigración, lengua y escuela}

La incorporación de alumnado extranjero a las aulas escolares es un fenómeno, ligado a la movilidad implicada en la globalización, que no va a detenerse. Al contrario, en los próximos años va a continuar creciendo y nuestra sociedad va ser aún más multicultural y plurilingüe. Por eso, las cuestiones que la educación intercultural coloca en primer plano y, entre ellas, las que se refieren al tratamiento lingüístico de la escuela van a ser cada vez más importantes en la reflexión y el debate educativo.

De hecho, los actores reales de la educación se han resistido durante muchos años a cuestionar el valor de la homogeneidad para la práctica educativa y la organización escolar. Probablemente, la razón más importante se relaciona con el funcionamiento de la sociedad industrial. La sociedad industrial era una sociedad vertical, jerarquizada en clases sociales, en la que todo el mundo sabía el lugar que ocupaba, así como las razones de dicho lugar. También se conocían bastante bien las fuentes de movilidad social y el valor de la educación. Por ejemplo, la actual sociedad española, una sociedad fundamentalmente de clases medias, es el resultado, entre otras cosas, de la Ley General de Educación, promulgada en 1972, que hacía la enseñanza obligatoria desde los 6 hasta los 14 años. Ahora bien, como todas las sociedades verticales y jerarquizadas, era una sociedad que, desde el punto de vista del mercado laboral, estaba segmentada y solicitaba requerimientos académicos distintos para cada uno de los segmentos. Por eso, un sistema educativo como el de Alemania, segregador ya desde la educación primaria y con itinerarios claramente diferenciados respecto al mercado laboral, tuvo tanto éxito y, sin embargo, hoy en día, está absolutamente obsoleto para las necesidades de la sociedad alemana (Allemann-Ghionda, 2006). Es evidente que la segregación y la homogeneidad van de la mano y, por tanto, a pesar del empuje de propuestas como la "enseñanza comprensiva" relacionada fundamentalmente con el mantenimiento de la equidad y la cohesión social, parece claro que, especialmente en la etapa de la enseñanza secundaria, las necesidades del mercado laboral de la sociedad industrial empujaban hacia la segregación y la homogeneidad (Tedesco, 1999).

Pero, en las actuales condiciones, el valor de la homogeneidad para la práctica educativa y la organización escolar pierde progresivamente enteros. Un buen ejemplo es el tratamiento de la diversidad lingüística en el contexto escolar. Históricamente, ante la presencia social de lenguas distintas en origen, la respuesta la ha dado "homogéneamente" la educación bilingüe. No obstante, cuando la presencia social de las lenguas en origen supera las 150 o más lenguas ¿cómo puede darse una respuesta homogénea? Es evidente que ni los programas de mantenimiento de la lengua familiar, ni los programas de inmersión lingüística, tal y como fueron diseñados a lo largo del Siglo XX, son válidos actualmente. ¿Podemos pensar en organizar 150 o más redes escolares según el grupo lingüístico del alumnado? El panorama resulta aterrador.

Por eso, desde la perspectiva de la lengua escolar, la cuestión es otra. No se trata de atender organizativamente la diversidad lingüística en origen, sino atender desde la práctica educativa y la organización escolar la diversidad del alumnado respecto al conocimiento de la lengua de la escuela. Cada vez será más normal que, en una misma aula de cualquier nivel de la educación infantil y la enseñanza obligatoria, conviva alumnado con un conocimiento enormemente 
diverso, diversidad desconocida hasta hace muy poco tiempo, de la lengua de la escuela. Así, podemos encontrar mezclados alumnado autóctono y alumnado extranjero escolarizados desde los 3 años, junto con una parte que se inició en la escuela a lo largo del parvulario y otra parte que lo hizo a lo largo de la enseñanza primaria y, si todavía hay sitio, puede llegar alumnado nuevo que no sabe nada de la lengua de instrucción. Es decir, da exactamente igual el número de lenguas que habla este alumnado, lo que es relevante para el trabajo educativo es la enorme dispersión del alumnado extranjero respecto a su conocimiento de la lengua de la escuela.

En este contexto, la homogeneidad tiene muy poco valor. A no ser que se aplique una política de "aulas-puente" y se mantenga al alumnado extranjero, como hemos visto, segregado durante años respecto al alumnado autóctono. ¿Alguien es capaz de defender una política educativa guiada por este patrón? ¿Podemos pensar en sus consecuencias para el futuro de la cohesión social? Además, también tiene muy poco valor el mantenimiento de la homogeneidad durante un tiempo más o menor corto, por ejemplo durante un año. ¿Qué gana y qué pierde educativamente el alumnado extranjero segregado durante un año del alumnado autóctono para aprender la lengua de la escuela? Gana poco como hemos visto y, sin embargo, pierde mucho. La lengua es un instrumento que regula intercambios sociales y, por tanto, no es la puerta de la integración. Al contrario, su aprendizaje y su dominio es el resultado de la integración (Carbonell, 2004). ¿Con quién aprende la lengua de la escuela el alumnado extranjero que permanece segregado junto con otros alumnos y otras alumnas que tampoco saben la lengua de la escuela? Más preguntas se quedan en el tintero. Todas ellas evidencian que el mantenimiento de la homogeneidad, en cualquiera de sus formas, deja cada vez más alumnado al margen de la educación escolar.

El inicio de los programas de inmersión lingüística fue un revulsivo en todas las sociedades que adoptaron este tipo de programas bilingües. Se trataba de que los y las escolares hicieran cosas con la lengua de la escuela sin saber dicha lengua. La idea vigotskiana según la cual el aprendizaje del habla remite a la idea de que hay que hablar cuando no se es capaz de hacerlo, de modo que si no se habla cuando no se es capaz de hablar nunca se llega a ser capaz de hablar (Bruner, 1983), guiaba la práctica educativa en la inmersión lingüística. Ello supuso un importantísimo movimiento de innovación educativa que, desgraciadamente, sólo alcanzó al parvulario y al primer ciclo de la educación primaria.

Actualmente, los retos educativos que planteó la inmersión lingüística se plantean nuevamente en la educación infantil y en toda la enseñanza obligatoria. Por eso, al igual que se hizo en el parvulario, allá por los años 80 , se debe de modificar la situación actual en el sentido de invertir el tiempo en que el profesorado habla y el alumnado escucha. Una gran parte de las cosas que pasan en las aulas se relaciona con dos premisas. Primero, una actividad unidireccional del profesorado hacia el alumnado en la que domina por encima de todo el habla del profesorado y la escucha del alumnado y, segundo, la primacía de la actividad individual incluso cuando el alumnado está organizado en grupos para trabajar. Hay muy pocas actividades, salvo honrosas excepciones, en las que las alumnas y los alumnos deban de hacer cosas juntos para resolver tareas complejas.

La modificación de esta situación consiste en colocar por delante el trabajo del alumnado en grupo; en modificar la actividad del profesorado de modo que pase a ser un actor que ofrezca las ayudas necesarias, en forma de informaciones y estrategias, para resolver tareas globales que tienen al alumnado como protagonista; en promover la interactividad entre el alumnado y, en consecuencia, el equilibrio entre exigencias y ayudas que, en el caso del lenguaje, son completamente necesarias si se desea que todo el alumnado progrese lingüísticamente; en 
hacer presente los diferentes "saberes” y “competencias” lingüísticas que el alumnado ya posee, valorándolas y mostrando la necesidad de que sean visibles para a la consecución colectiva de las tareas propuestas. En fin, una manera diferente de hacer, en la que no necesariamente se discute el método didáctico más o menos pertinente para conseguir un objetivo de enseñanza y aprendizaje, sino que coloca en primer plano la diversidad real respecto al conocimiento de la lengua de la escuela como fuente del trabajo cooperativo.

\section{Notas}

"Ignasi Vila es Catedrático de Universidad del área de Psicología Evolutiva y de la Educación de Universidad Girona. Su campo de trabajo ha abarcado la adquisición del lenguaje, la educación bilingüe, la didáctica de las lenguas, etc. Otros ámbitos de interés relacionados son la psicología sociocultural o las relaciones familia-comunidad.

${ }^{1}$ Esta fórmula no ha sido nunca utilizada por la Comunidad Autónoma Vasca y recientemente ha sido abandonada por la Generalitat de Cataluña

${ }^{2}$ Las excepciones son Canadá, Italia, Nueva Zelanda, Australia, Irlanda e Islandia.

${ }^{3}$ De los países con un elevado porcentaje de extranjeros, se ha de exceptuar Canadá, Australia y Nueva Zelanda

${ }^{4}$ En algunas provincias como Ontario la población de origen extranjero representa la mitad de la población.

\section{Referencias}

Ajuntament de Barcelona (2005). La població estrangera a Barcelona. Gener 2005. Barcelona: Estadístiques de l'Ajuntament de Barcelona.

ALLEMANN-GHIONDA, C. (2006). Intercultural education in Germany: accepting diversity while cultivating differences? Comunicación al Seminario Internacional "Educating Immigrant Pupils: Approaches and policies challenging educational integration and equal opportunities for all". Fundació Jaume Bofill. Barcelona.

ArenY, M. \& Vial, S. (2005). Estudi del nivell de coneixement del català i del castellà de l'alumnat immigrat en acabar el Cicle Inicial d'Educació Primària. Comunicació al Tercer Simposi sobre l'ensenyament del català a no-catalanoparlants. Vic (Barcelona), septiembre.

Bel, A., Serra, J. M. \& Vila, I. (1992). El coneixement de llengua catalana a Gè, 7è i gè d'EGB. Documento no publicado. Barcelona: ICE de la Universitat de Barcelona.

Broeder, P. y Mijares, L. (2003). Plurilingüismo en Madrid. Madrid: Comunidad de Madrid/Ministerio de Educación, Cultura y Deporte.

BRunER, J. S. (1983). Child's Talk. Nueva York: Norton.

CARbonell, F. (2004). Educar en temps d'incertesa. Palma de Mallorca: Lleonard Muntaner.

COELHO, E. (1998). Teaching and Learning in Multicultural Schools: An Integrated Approach. Clevedon, UK: Multilingual Matters.

Coelho, E. (2003). Adding English: A Guide to Teaching in Multilingual Classrooms. Toronto: Pippin Publishing.

COLLIER, V. P. (1987). Age and rate of acquisition of second language for academic purposes. TESOL Quarterly, 21, 617-641.

Cummins, J. (1979). Linguistic interdependence and the educational development of bilingual children. Review of Educational Research, 49, 222-251.

CumMINS, J. (1981) Age on arrival and immigrant second language learning in Canada: A reassessment. Applied Linguistics, 1, 132149.

Cummins, J. (2001). Negotiating identities: education for empowerment in a diverse society. Los Angeles, CA: California Association for Bilingual Education.

Cummins, J. (2002). Lenguaje, poder y pedagogía. Madrid: Morata.

Ferrer, F., Ferrer, G. \& CAStel, J.L. (2006). Les desigualtats educatives a Catalunya: PISA 2003. Barcelona: Fundació Jaume Bofill.

ForTIN, L. (2006). L'intégration scolaire des élèves immigrants au Québec entre la classe d'accueil el la classe ordinaire: un aperçu quant aux pratiques, aux actions et aux perspectives. Comunicación al Seminario Internacional "Educating Immigrant Pupils: Approaches and policies challenging educational integration and equal opportunities for all". Fundació Jaume Bofill. Barcelona, marzo.

FullanA, J., BeSAlÚ, X. \& VILÀ, M. (2003). Alumnes d'origen africà a l'escola. Girona: CCG Edicions.

GANDARA, P. (1999). Review of research on instruction of limited English proficiency students: A report to the California legislatura. Santa Bárbara: University of California, Linguistic Minority Research Institute.

Hakuta, K., Butler, Y. G. \& Witt, D. (2000). How Long Does It Take English Learners to Attain Proficiency? Santa Barbara, CA: University of California Linguistic Minority Research Institute.

Huguet, A. \& NAvarro, J. L. (2005). Inmigrantes en la escuela. Una revisión de estudios sobre las relaciones entre rendimiento escolar e inmigración. En D. Lasagabaster \& J. M. Sierra (Eds.), Multilingüismo, competencia lingüística y nuevas tecnologías (pp. 5374). Barcelona: Horsori

INECSE (2006). Evaluación de la Educación Primaria 2003. Madrid: Ministerio de Educación y Ciencia.

IZQuierdo, A. (2002). La educación errante. En AA.VV., La sociedad. Teoría e investigación empírica (pp. 207-230). Madrid: Centro de Investigaciones Sociales.

KLESMER, H. (1994). Assessment and teacher perceptions of ESL students achievement. English Quarterly, 26 (3), 8-11.

KRASHEN, S. (1985). The Input Hypothesis: issues and implications. Nueva York: Longman.

MARUNY, L. \& MolinA, M. (2000). Adquisició del català i competència comunicativa en alumnes d'origen marroquí a l'ensenyament obligatori. Competència discursiva, competència lectora, competència escriptora. Comunicació al Seminari Interculturalitat, educació i llengües. Girona: Comissions Obreres. 
MC ANDREw, M. (2001). Immigration et diversité à l'école. Montréal: Les Presses de l'Univrsité de Montréal.

MC ANDREw, M. (2002). La loi 101 en milieu scolaire: impacts et résultats. Revue d'Aménagement Linguistique, Hors série, 69-83.

McLaughlin, B. (1985). Second language Acquisition in Childhood: Vol. 2, School-Age Children. Hillsdale, NJ: Lawrence Erlbaum Associates.

NADAL, J. M. (2006). La llengua sobre el paper. Girona: CCG Edicions.

NAvARro, J. L. \& Huguet, Á. (2005). El conocimiento de la lengua castellana en alumnado inmigrante escolarizado en Primero de ESO. Un estudio empírico. Madrid: CIDE.

OCDE (2002). Conocimientos y destrezas para la vida. Primeros resultados del Proyecto PISA 2000. Madrid: Ministerio de Educación y Ciencia.

RAMírez, J. D. (1992). Executive summary. Bilingual Research Journal, 16, 1-62.

SiguAN, M. (1998). La escuela y los inmigrantes. Barcelona: Paidós.

Shohamy, E. (1999). Unity and Diversity in Language Policy. Comunicación en la AILA Conference. Tokio, agosto.

TeDesco, J. C. (1999). Els fenòmens de segregació i exclusió social del coneixement i de la informació. En AA. VV., Per una ciutat comprometa amb l'educació (Vol. II, pp. 19-31). Barcelona: Institut d'Educació de l'Ajuntament de Barcelona.

THOMAS, W. P. \& COLLIER, V. (1997). School effectiveness for language minority students. Washington, DC: National Clearinghouse for Bilingual Education.

VILA, I. (2000). Inmigración, educación y lengua propia. En AA.VV., La inmigración extranjera en España. Los retos educativos (pp. 145-166). Fundación La Caixa: Barcelona.

VILA, I. (2005a). ¿Nivel sociocultural o desconocimiento de la lengua? Perspectivacep, 8, 23-54.

Vila, I. (2005b). Actualidad y perspectivas de la educación bilingüe en el Estado español. En X. P. Rodríguez-Yánez, A. M. Lorenzo Suárez \& F. Ramallo (Eds.), Bilingualism and Education: From the Family to School (pp. 339-352). Muenchen: Lincom Europa.

Vila, I., Huguet, Á. \& Navarro, J. L. (2005). Ethnical origin, time of residence and knowledge of the school language. Comunicación al Simposio Invitado "Bilingualism in children exposed to Spanish and another language in Spain". XIIth European Conference on Developmental Psychology. La Laguna, agosto.

VILA, I. \& SiQuÉs, C. (2006a). Infancia estrangera i coneixement de la llengua de l'escola. Articles de Didàctica de la Llengua i la Literatura, 38, 29-37.

VILA, I. \& SIQUÉs, C. (2006b). Educación, pluralidad lingúística y construcción de la identidad. Comunicación al MIDP Symposium Multilingualism and Exclusion. Bloemfontein (Sudáfrica), abril.

VILA, I. \& SIQUÉs, C. (en prensa). Lengua propia y lengua de la escuela: implicaciones educativas en la escolarización de la infancia extranjera. En I. Márquez (Ed.), Respuestas a la exclusión: políticas de inmigración, interculturalidad y mediación. Madrid: Dykinson. 